\title{
How to Prevent and Manage Hospital-Based Infections During Coronavirus Outbreaks: Five Lessons from Taiwan
}

\author{
C Jason Wang, MD, PhD ${ }^{1,2^{*}}$, Henry Bair ${ }^{3}$, and Ching-Chuan Yeh, MD, MPH ${ }^{4}$
}

'Department of Pediatrics, Department of Medicine, and Department of Health Research and Policy, Stanford University School of Medicine, Stanford, California; ${ }^{2}$ The New School for Leadership in Health Care, Koo Foundation Sun Yat-Sen Cancer Center, Taipei, Taiwan; ${ }^{3}$ Stanford University School of Medicine, Stanford, California; ${ }^{4}$ Department of Public Health, Tzu-Chi University, Hualien, Taiwan.

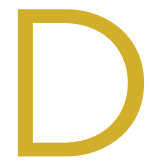

uring the severe acute respiratory syndrome (SARS) outbreak in 2003, Taiwan reported 346 confirmed cases and 73 deaths. ${ }^{1}$ Of all known infections, 94\% were transmitted inside hospitals. Nine major hospitals were fully or partially shut down, and many doctors and nurses quit for fear of becoming infected. The Taipei Municipal Ho-Ping Hospital was most severely affected. Its index patient, a 42-yearold undocumented hospital laundry worker who interacted with staff and patients for 6 days before being hospitalized, became a superspreader, infecting at least 20 other patients and 10 staff members. ${ }^{2,3}$ The entire 450-bed hospital was ordered to shut down, and all 930 staff and 240 patients were quarantined within the hospital. The central government appointed the previous Minister of Health as head of the Anti-SARS Taskforce. Ultimately the hospital was evacuated; the outbreak resulted in 26 deaths. ${ }^{2}$ Events surrounding the hospital's evacuation offer important lessons for hospitals struggling to cope with the COVID-19 pandemic, which has been caused by spread of a similar coronavirus.

\section{LESSON 1: DIAGNOSIS}

Flexibility about case definition is important, as is use of clinical criteria for diagnosis when reliable laboratory tests are not available.

The laundry worker of Ho-Ping Hospital was initially misdiagnosed with infectious enteritis, which delayed proper management and, crucially, isolation from other patients. The low index of suspicion for SARS reflected the initial World Health Organization diagnostic criteria for SARS, which included travel to or residence in an area with recent local transmission of SARS within 10 days of symptom onset. ${ }^{4}$ The laundry worker did not have a recent travel history. ${ }^{3}$ Additionally, SARS manifested as a lower respiratory tract infection, so many patients were hospitalized for pneumonia before being diagnosed with SARS. Similarly, the Wuhan Municipal Health Commission initially issued diagnostic criteria for COVID-19 that, in addition to fever and symptoms of respiratory infections, emphasized direct exposure to the Huanan Seafood Wholesale Market. ${ }^{5}$ As a result, many cases of COVID-19 were not identified.

*Corresponding author: C Jason Wang, MD, PhD; Email: cjwang1@stanford. edu; Telephone: 650-736-0403; Twitter: @jasonwang_md.

Published online first May 20, 2020

Received: April 12, 2020 Revised: April 15, 2020 Accepted: April 22, 2020

(c) 2020 Society of Hospital Medicine DOI 10.12788/jhm.3452
Diagnosing SARS was challenging. Early symptoms such as fever and malaise were nonspecific. Polymerase chain reaction tests, although available, were unreliable especially in early stages of the disease and had a high false-negative rate. As cases of SARS increased rapidly, Taiwan began using fever alone for early detection. ${ }^{6}$ Patients and hospital staff received temperature measurements twice daily. Despite the late start to SARS screening, the fever criterion identified many suspected patients, which ensured widespread detection and containment.

For COVID-19, symptoms such as fever, dry cough, and shortness of breath can be used as clinical criteria to triage patients for quarantine in endemic areas when reliable diagnostic tests are not readily available, but all frontline clinical staff should receive daily temperature checks and/or COVID-19 tests, if available, to protect their families and the public.

\section{LESSON 2: COORDINATION}

Ineffective coordination between central and local governments can delay response, but this can be remedied.

During the SARS outbreak, the Taipei City Government and the Taiwan central government were controlled by opposing political parties. Responses to SARS were initially impeded by political skirmishes, which hindered implementation of policies regarding criteria for diagnosis, tracking of suspected cases and their contacts, duration of quarantine, and allocation of resources and facilities for confirmed cases. To avoid further delays, the central government acted swiftly to create the nonpartisan Anti-SARS Taskforce and appointed leaders who could work cordially with both local and central government agencies. To help to deal with the crisis, the central government also designated a new Minister of Health, an epidemiologist, who became the first nonphysician to hold this position.

\section{LESSON 3: EVACUATION}

\section{Treatment in place vs evacuation during hospital infections} is a critical decision.

The surge of SARS cases at Ho-Ping Hospital led to confusion and panic among patients and hospital staff. Whether to treat its SARS patients on site or to evacuate the hospital was a complex decision and reflected many concerns, including the following: How many wards had been infected? Was there sufficient equipment (eg, respirators) to monitor or treat infected patients? How many isolation beds were available? How many hospital staff were already infected and quarantined? Were they in different wards? Were there neighboring facilities (eg, 
hospitals, military camps, dorms) available for quarantine?

If a hospital has sufficient capacity to isolate persons under investigation and to treat confirmed patients, on-site treatment is possible. However, evacuation should be considered when there is widespread infection involving different hospital wards and hospital staff. In such cases, patients should be transferred to different facilities based on clinical severity: $\mathrm{Pa}$ tients with new onset fever or respiratory symptoms but who are relatively healthy should be sent to community or regional hospitals with isolation rooms for monitoring; sicker infected patients should be sent to medical centers; and other hospitalized patients (eg, admitted for heart failure) without infection risk or symptoms should seek care elsewhere.

So far, there has been only one instance of hospital-based COVID-19 infections in Taiwan, and the spread of infection was quickly contained within one ward. All nine confirmed cases (including the index patient, one patient in the same ward but a different room, three nurses, one laundry worker, and three members of patients' families) and their known contacts were identified, then isolated or quarantined individually. Because the affected hospital is part of a complex with more than 3,000 beds, it was big enough to accommodate all infected patients and no evacuation measures were needed. To further reduce potential nodes in the chain of transmission, interns and many other healthcare workers were temporarily relieved of their duties, elective surgeries were canceled, and hospital visitation was limited to immediate family members. The clear communication of intervention measures ensured rapid cooperation and staved off both social panic and further spread of the disease.

\section{LESSON 4: PATIENT FLOW}

\section{Hospitals should establish different flows for different pa- tients.}

Having learned from the SARS experience in 2003, hospitals in Taiwan have designated specific pathways to manage patient flow during the COVID-19 outbreak, in addition to checking all patients for travel history and fever: Patients with fever were quickly triaged to a designated fever clinic so they did not mingle with other patients, patients visiting the hospital to

\section{References}

1. Hsieh YH, King CC, Chen CWS, et al. Quarantine for SARS, Taiwan. Emerg Infect Dis. 2005;11(2):278-282. https://doi.org/10.3201/eid1102.040190.

2. From the Centers for Disease Control and Prevention. Severe Acute Respiratory Syndrome - Taiwan, 2003. JAMA. 2003;289(22):2930-2932. https://doi. org/10.1001/jama.289.22.2930

3. McNeil DG. The SARS epidemic: the virus; most Taiwan SARS cases spread by one misdiagnosis. New York Times. May 8, 2003. https://www.nytimes. com/2003/05/08/world/the-sars-epidemic-the-virus-most-taiwan-sars-casesspread-by-one-misdiagnosis.html. Accessed March 28, 2020.

4. Hui DSC, Chan MCH, Wu AK, Ng PC. Severe acute respiratory syndrome obtain chronic disease medications were directed to a "drivethrough" lane, patients needing emergent care went through the emergency department, all other regular outpatients were seen in outpatient departments, and visitors of patients were restricted to one visitor per patient at a given time.

\section{LESSON 5: ORGANIZATION}

\section{Healthcare providers should be organized into blocks and modular teams to avoid hospital-wide infection.}

After SARS, Taiwan learned that one way to reduce the spread of something like COVID-19 among healthcare providers and from providers to patients is to divide providers' work areas into discrete blocks and organize providers into modular teams. This approach was inspired by the design of watertight compartments in ships: Should the hull be breached, flooding is restricted to the breached compartments. Under this organizational strategy, movements of physicians and nurses would be restricted to their designated locations: They would be routinely exposed only to other staff and patients within their division. Doctors and nurses would be asked to practice in modular teams within their blocked locations, reducing the likelihood that infection in one team would spread to another, which could lead to hospital-wide infections. Movement of senior hospital executives would be similarly restricted. Common areas such as cafeterias where people mingle would be closed. Owing to these stringent initiatives, aside from the hospitalbased infection mentioned in Lesson 3, no other hospitalbased infections have been reported in Taiwan so far.

\section{CONCLUSION}

Lessons from previous hospital-based coronavirus infections can be used to minimize future infections.

\section{Acknowledgments}

The authors would like to thank Dr Lee Ming-Liang, former Health Minister of Taiwan and director of that central government's Anti-SARS Taskforce during the 2003 outbreak, for providing valuable recommendations to this work.

Disclosures: The authors reported having nothing to disclose.

(SARS): epidemiology and clinical features. Postgrad Med J. 2004;80(945):373381. https://doi.org/10.1136/pgmj.2004.020263.

5. Yang DL. Wuhan officials tried to cover up covid-19 - and sent it careening outward. Washington Post. March 10, 2020. https://www.washingtonpost. com/politics/2020/03/10/wuhan-officials-tried-cover-up-covid-19-sent-it-careening-outward/. Accessed March 28, 2020.

6. Lin EC, Peng YC, Hung Tsai JC. Lessons learned from the anti-SARS quarantine experience in a hospital-based fever screening station in Taiwan. Am J Infect Control. 2010;38(4):302-307. https://doi.org/10.1016/j. ajic.2009.09.008. 